cond-mat/0006234

\title{
Nodal Liquids and Duality
}

\author{
Nick E. Mavromatos and Sarben Sarkar \\ Department of Physics, King's College London, \\ Strand, London WC2R 2LS, United Kingdom.
}

\begin{abstract}
Using a $\mathrm{SU}(2) \times \mathrm{U}(1)$ gauge theory for a $\mathrm{t}-\mathrm{J}$ model around a node of the Fermi surface, we discuss patterns of dynamical symmetry breaking, which may lead to a pseudogap phase and to the appearance of narrow one-dimensional spatial structures, induced by the presence of holes. A possible connection with stripe phases is briefly discussed by passing to an appropriate dual theory. We discuss confinement properties of spinons and holons and derive the spectrum of the (gapful) physical excitations which are composites of holons corresponding to 'mesons', and composites of spinons corresponding to 'baryons'.
\end{abstract}

Strong electron correlations are regarded generally as an important ingredient of a theory for high temperature superconductivity. There has been a great deal of ingenuity in theoretical approaches and this has been concentrated on the most appropriate way of incorporating constraints in terms of 'particle' occupancy at sites of the underlying lattice. Symmetry, both global and local and both in the Wigner-Weyl mode or the Nambu-Goldstone mode have been shown to be essential ingredients. However the exact symmety group and the representation and nature of any symmetry breaking forms are the questions which need to be settled.

In this note we will put forward a candidate for this symmetry [1]:

$$
G \simeq \mathrm{SU}(2) \times \mathrm{U}(1)
$$

where $G$ is a local symmetry. The $\mathrm{SU}(2)$ symmetry is present in a Nambu-Goldstone form and only a compact $\mathrm{U}_{\tau}(1)$ associated with the $\tau_{3}$ generator of $\mathrm{SU}(2)$ remains unbroken. In the physical $(2+1)$-dimensional model [1] the statistical gauge symmetry $\mathrm{U}(1)$ appears in a gauge fixed form, and in fact is associated with exotic statistics of the relevant excitations. This basic symmetry breaking which is achieved dynamically [1] leads to a gauge-invariant mass gap for charge degrees of freedom (the pseudogap) and also to 'one dimensional' structures which we suggest are related to the insulating stripe phases which are of current interest in underdoped cuprates. In addition there is a global $\mathrm{SU}(2)$ symmetry related to the spin which in the gauge theory approach will be associated with a 'custodial' SU(2) global symmetry, as we shall discuss at the end.

Our aim is, starting from a reasonable microscopic Hamiltonian, $H$, to form a continuum field theory for the low energy excitations of the cuprates. Such a model belongs to a class of $t-J$ models with additional interactions [1] [2]. At half-filling $H$ reduces to the Heisenberg Hamiltonian for which adding a spin-up particle is the same as removing a spin-down particle owing to the constraint of single particle occupancy. This in turn leads to a 'hidden' SU(2) gauge symmetry of the Heisenberg model. In the presence of holes this no longer holds, and the theory has a $\mathrm{U}(1)$ gauge symmetry. Usually there is a 'discontinuity' in the formulation of the problem at half-filling and away from half-filling. In the former there is a local SU(2) symmetry while in the latter the $\mathrm{SU}(2)$ formulation is totally replaced with a $\mathrm{U}(1)$ local symmetry from the outset. In our case we have the symmetry group $G$ which reduces to $\mathrm{SU}(2)$ at half-filling while away from half-filling, owing to strong gauge fluctuations associated with the $\mathrm{U}(1)$ factor in $G$, the $\mathrm{SU}(2)$ 
is dynamically broken down to $\mathrm{U}_{\tau}(1)$, i.e. the system decides about the local symmetry given a maximal $G$-symmetry.

In the two-dimensional Heisenberg model Affleck et al [3] noted that

$$
\underline{S}_{i}=\operatorname{Tr}\left(\chi_{i}^{\dagger} \chi_{i} \underline{\sigma}^{\mathrm{T}}\right)
$$

where, in terms of $c_{1}$ and $c_{2}$ (the annihilation operators for up and down electrons),

$$
\chi=\left(\begin{array}{rr}
c_{1} & c_{2} \\
c_{2}^{\dagger} & -c_{1}^{\dagger}
\end{array}\right)
$$

and so there is a local SU(2) symmetry $\chi_{i} \longrightarrow h_{i} \chi_{i}, \quad h_{i} \in \mathrm{SU}(2)$. In all the above the index $i$ labels the lattice sites and $\underline{\sigma}$ is the vector of Pauli-matrices.

Away from half-filling we make the ansatz [1]

$$
\chi=\left(\begin{array}{rr}
\psi_{1} & \psi_{2} \\
\psi_{2}^{\dagger} & -\psi_{1}^{\dagger}
\end{array}\right)\left(\begin{array}{rr}
z_{1} & -\bar{z}_{2} \\
z_{2} & \bar{z}_{1}
\end{array}\right)
$$

where $\psi_{\alpha}, z_{\alpha}$ with $\alpha \in\{1,2\}$ are fermions and bosons respectively. The $\psi_{\alpha}$ describe charge degrees of freedom and the $z_{\alpha}$ describe the spin degrees of freedom. The index $\alpha$ is related to the underlying bipartite (antiferromagnetic) lattice structure. At a site the original formulation had two degrees of freedom: the spin-up hole and the spin-down hole. The above spin-charge separation ansatz should have constraints and symmetries which reduce the number of physical degrees of freedom to two. One constraint is that $c_{\alpha}$ should obey canonical commutation relations. On assuming canonical commutation/anti-commutation relations for $z_{\alpha}$ and $\psi_{\alpha}$ we find that the canonical anticommutation relations for the $c$ operators are satisfied on physical states provided the following constraints at each site $i$ hold [1]:

$$
\psi_{1, i} \psi_{2, i}=0=\psi_{2, i}^{\dagger} \psi_{1, i}^{\dagger}, \sum_{\beta=1,2}\left(\bar{z}_{i, \beta} z_{i, \beta}+\psi_{\beta, i}^{\dagger} \psi_{\beta, i}\right)=1
$$

These relations are single occupancy constraints and imply that our formulation is suitable in the limit of strong correlations. It will be convenient to work in the functional integral framework where the $\psi$ are Grassmann variables. If we count the degrees of freedom in $\chi$, $\operatorname{deg} \chi$, we have $\operatorname{deg} \chi=4+4$, where 4 counts the Grassmann degrees of freedom and 4 is the number of real degrees of freedom in $z_{1}, z_{2}, \bar{z}_{1}$ and $\bar{z}_{2}$.

Just as in the Heisenberg case there is an $\mathrm{SU}(2)$ symmetry

$$
\begin{array}{rrr}
\Psi_{i} \longrightarrow \Psi_{i} h_{i} & \text { where } & \Psi=\left(\begin{array}{rr}
\psi_{1} & \psi_{2} \\
\psi_{2}^{\dagger} & -\psi_{1}^{\dagger}
\end{array}\right) \\
Z_{i} \longrightarrow h_{i}^{\dagger} Z_{i} & \text { where } & Z=\left(\begin{array}{rr}
z_{1} & -\bar{z}_{2} \\
z_{2} & \bar{z}_{1}
\end{array}\right)
\end{array}
$$

where $h_{i} \in \mathrm{SU}(2)$. However, there is also a dynamical $\mathrm{U}(1)$ gauge symmetry acting on the $\Psi$ fields, which is due to phase frustration from holes moving in a spin background. Some arguments to justify this from a microscopic point of view have been given in reference [1]. Consequently, this symmetry is associated with exotic statistics of the pertinent excitations [1], which is an exclusive feature of the planar spatial geometry. The existence of these gauge symmetries together with the constraints reduce the effective number of degrees of freedom to the physical degrees of freedom of the system. The ansatz gives us a maximal symmetry $G$. The dynamics will be specified by a Hamiltonian which will determine any spontaneous symmetry breaking that occurs.

As a generic model we will consider a generalized $\mathrm{t}-\mathrm{J}$ model which is an effective singlebond model derived from a more realistic 5-bond model [2]. This is in terms of the parameters $\left\{t, t^{\prime}, t^{\prime \prime}, J, V\right\}$ where $t, t^{\prime}$, and $t^{\prime \prime}$ are nearest-neighbour, next nearest-neighbour and third nearestneighbour hoppings respectively, $J$ is the Heisenberg antiferromagnetic interaction and $V$ is a 
static attractive nearest-neighbour interaction. It turns out that, under certain circumstances, the presence of $V$ allows the theory in the continuum limit to show dynamical supersymmetry between spinon and holon degrees of freedom as has been discussed in ref. [2].

The Heisenberg term can be written in terms of a Hubbard-Stratonovich field, $\Delta_{i j}$, as

$$
-\frac{J}{8} \sum_{\langle i j\rangle} \operatorname{Tr}\left(\chi_{i} \chi_{j}^{\dagger} \chi_{j} \chi_{i}^{\dagger}\right)=\sum_{\langle i j\rangle} \operatorname{Tr}\left[\frac{8}{J} \Delta_{i j}^{\dagger} \Delta_{j i}+\left(\chi_{i}^{\dagger} \Delta_{i j} \chi_{j}+\text { h.c. }\right)\right] .
$$

The hopping part of the Hamiltonian can be written as

$$
\begin{aligned}
-\sum_{\langle i j\rangle} t_{i j} c_{\alpha, i}^{\dagger} c_{\alpha, j}= & -\sum_{\langle i j\rangle} t_{i j}\left(\chi_{i, \alpha \gamma}^{\dagger} \chi_{j, \gamma \alpha}+\chi_{i, \alpha \gamma}^{\dagger}\left(\sigma_{3}\right)_{\gamma \beta} \chi_{j, \beta \alpha}\right)= \\
& =-\sum_{\langle i j\rangle} t_{i j}\left(\bar{Z}_{i, \beta \kappa} \Psi_{i, \kappa \alpha}^{\dagger} \Psi_{j, \alpha \gamma} Z_{j, \gamma \beta}+\bar{Z}_{i, \beta \kappa} \Psi_{i, \kappa \alpha}^{\dagger}\left(\sigma_{3}\right)_{\alpha \lambda} \Psi_{j, \lambda \gamma} Z_{j, \gamma \beta}\right) .
\end{aligned}
$$

The global SU(2) spin symmetry, mentioned above, is explicitly given by $Z_{i} \rightarrow Z_{i} h$ (and equivalently $\left.\chi_{i} \rightarrow \chi_{i} h\right)$ where $h \in \mathrm{SU}(2)$ is a group element.

In the Hartree-Fock approximation we obtain the Hamiltonian

$$
\begin{aligned}
H_{\mathrm{HF}}=\sum_{\langle i j\rangle} \operatorname{Tr}\left[\frac{8}{J} \Delta^{\dagger} i j \Delta_{j i}+\left(-t_{i j}(1\right.\right. & \left.\left.\left.+\sigma_{3}\right)+\Delta_{i} j\right) \Psi_{j}^{\dagger}\left\langle Z_{j} \bar{Z}_{i}\right\rangle \Psi_{i}\right]+ \\
& +\sum_{\langle i j\rangle} \operatorname{Tr}\left[\bar{Z}_{i}\left\langle\Psi_{i}^{\dagger}\left(-t_{i j}\left(1+\sigma_{3}\right)+\Delta_{i} j\right) \Psi_{j}\right\rangle Z_{j}+\text { h.c. }\right] .
\end{aligned}
$$

Using the $G$-symmetry (11) of the ansatz we can write in the presence of gauge fixing

$$
\left\langle Z_{j} \bar{Z}_{i}\right\rangle \equiv\left|A_{1}\right| \mathcal{R}_{i j} U_{i j},\left\langle\Psi_{i}^{\dagger}\left(-t_{i j}\left(1+\sigma_{3}\right)+\Delta_{i} j\right) \Psi_{j}\right\rangle \equiv\left|A_{2}\right| \mathcal{R}_{i j} U_{i j} .
$$

where $\mathcal{R} \in \mathrm{SU}(2)$ and $U \in \mathrm{U}(1)$ are group elements. The fact that apparently gauge non-invariant correlators are non-zero on the lattice is standard in gauge theories, and does not violate Elitzur's theorem [5], precisely due to the above-mentioned gauge-fixing procedure, which is done prior to any computation. The amplitudes $\left|A_{1}\right|$ and $\left|A_{2}\right|$ are considered frozen which is a standard assumption in the gauge theory approach to strongly correlated electron systems [3].

By standard arguments the low energy lattice action for the fermion part becomes

$$
S_{\mathrm{F}}=\frac{1}{2} \kappa^{\prime} \sum_{i, \mu}\left(\overline{\hat{\Psi}}_{i} \gamma_{\mu} \mathcal{R}_{i, \mu} U_{i, \mu} \hat{\Psi}_{i+\mu}+\overline{\hat{\Psi}}_{i+\mu} \gamma_{\mu} \mathcal{R}_{i, \mu} U_{i, \mu} \hat{\Psi}_{i}\right)
$$

where $\hat{\Psi}_{i, \alpha}$ is a Nambu-Dirac two-component spinor for each 'colour' $\alpha \in\{1,2\}$. The $\mathcal{R}$ and $U$ matrices act on the colour matrices whereas the $2 \times 2 \gamma$ matrices act on the two components of $\hat{\Psi}_{i, \alpha}$. The components of $\hat{\Psi}$ are linearly related to those of $\Psi$ Moreover, we have kinetic (i.e. Maxwell) terms for the gauge link variables in the form of plaquette terms in the lattice action:

$$
S_{\mathrm{G}}=\sum_{p}\left[\beta_{2}\left(1-\operatorname{Tr} \mathcal{R}_{p}\right)+\beta_{1}\left(1-\operatorname{Tr} U_{p}\right)\right]
$$

where $p$ denotes plaquettes, the $\beta_{i}$ are inverse couplings, $\beta_{2} \equiv \beta_{\mathrm{SU}(2)} \propto 1 / g^{2}, \beta_{1} \equiv \beta_{\mathrm{U}(1)}$, and $\mathcal{R}_{p}, U_{p}$ are a product of the link variables over the plaquette $p$. It is important to notice for our purposes that, as a result of the gauge-fixed form of the $\mathrm{U}(1)$ factor in $G$ in the spinon sector, there is no plaquete term for the $\mathrm{U}(1)$ field, which implies $\beta_{1}=0$ and hence we are in the strong coupling limit [1]. The coupling $\beta_{2}$ is large [2].

We will find it useful to give the explicit representation of the global $G$-symmetry whose gauging produces $S_{\mathrm{G}}$. On writing

$$
\tilde{\Psi}=\left(\begin{array}{c}
\hat{\Psi}_{1} \\
\hat{\Psi}_{2}
\end{array}\right)
$$


the generators of $G$ are given by

$$
\left\{\mathbf{1}_{4},\left(\begin{array}{cc}
0 & \mathbf{1}_{2} \\
\mathbf{1}_{2} & 0
\end{array}\right), i\left(\begin{array}{cc}
0 & \mathbf{1}_{2} \\
-\mathbf{1}_{2} & 0
\end{array}\right),\left(\begin{array}{cc}
\mathbf{1}_{2} & 0 \\
0 & -\mathbf{1}_{2}
\end{array}\right)\right\}
$$

Let us consider some relevant multiplets of this group at any site $j$. The bilinears

$$
\phi_{1}=-i\left(\overline{\hat{\Psi}}_{1} \hat{\Psi}_{2}-\overline{\hat{\Psi}}_{2} \hat{\Psi}_{1}\right), \phi_{2}=\overline{\hat{\Psi}}_{1} \hat{\Psi}_{2}+\overline{\hat{\Psi}}_{2} \hat{\Psi}_{1}, \phi_{3}=\overline{\hat{\Psi}}_{1} \hat{\Psi}_{1}-\overline{\hat{\Psi}}_{2} \hat{\Psi}_{2}
$$

form an adjoint representation of $\mathrm{SU}(2)$ [1]. There is also a vector adjoint representation

$$
\begin{aligned}
& \left(\mathcal{A}_{\mu}\right)_{1}=i\left(\overline{\hat{\Psi}}_{1} \tilde{\sigma_{\mu}} \hat{\Psi}_{2}-\overline{\hat{\Psi}}_{2} \tilde{\sigma}_{\mu} \hat{\Psi}_{1}\right),\left(\mathcal{A}_{\mu}\right)_{2} \quad=\overline{\hat{\Psi}}_{1} \tilde{\sigma}_{\mu} \hat{\Psi}_{2}+\overline{\hat{\Psi}}_{2} \tilde{\sigma}_{\mu} \hat{\Psi}_{1}, \\
& \left(\mathcal{A}_{\mu}\right)_{3}=\overline{\hat{\Psi}}_{1} \tilde{\sigma_{\mu}} \hat{\Psi}_{1}-\overline{\hat{\Psi}}_{2} \tilde{\sigma_{\mu}} \hat{\Psi}_{2}
\end{aligned}
$$

and two singlets

$$
\mathcal{S}_{4}=\overline{\hat{\Psi}}_{1} \hat{\Psi}_{1}+\overline{\hat{\Psi}}_{2} \hat{\Psi}_{2},\left(\mathcal{S}_{\mu}\right)_{4}=\overline{\hat{\Psi}}_{1} \tilde{\sigma}_{\mu} \hat{\Psi}_{1}+\overline{\hat{\Psi}}_{2} \tilde{\sigma}_{\mu} \hat{\Psi}_{2}
$$

where $\tilde{\sigma_{0}}=-i \sigma_{3}, \tilde{\sigma_{1}}=\sigma_{1}$ and $\tilde{\sigma_{2}}=\sigma_{2}$.

Now we will consider spontaneous symmetry breaking in $G$ due to the interactions. Since we are at the strong coupling limit of $\mathrm{U}(1)$, where $\beta_{1}=0$, the $\mathrm{U}(1)$ gauge field can be integrated out exactly to give [1]

$$
\int \mathfrak{D R} \mathfrak{D} \hat{\Psi} \mathfrak{D} \hat{\Psi} \exp \left(-S_{\text {eff }}\right)
$$

where

$$
\begin{aligned}
S_{\text {eff }} & =\beta_{2} \sum_{p}\left(1-\operatorname{Tr} \mathcal{R}_{p}\right)+\sum_{i, \mu} \ln I_{0}\left(\sqrt{y_{i \mu}}\right), \\
y_{i \mu} & =-\kappa^{2} \operatorname{Tr}\left(M^{(i)}\left(-\gamma_{\mu}\right) \mathcal{R}_{i \mu} M^{(i+\mu)} \gamma_{\mu} \mathcal{R}_{i \mu}^{\dagger}\right)
\end{aligned}
$$

and

$$
M^{(i)}=\sum_{a=1}^{3} \phi_{a}(i) \sigma_{a}+\mathcal{S}_{4}(i) \mathbf{1}+i\left(\left(\mathcal{S}_{\mu}\right)_{4}(i) \gamma^{\mu}+\sum_{a=1}^{3}\left(\mathcal{A}_{\mu}\right)_{a}(i) \gamma^{\mu} \sigma_{a}\right) .
$$

Owing to the Grassmann content of $M$,

$$
-\ln I_{0}^{\operatorname{tr}}\left(2 \sqrt{y_{i \mu}}\right)=-y_{i \mu}+\frac{1}{4} y_{i \mu}^{2}-\frac{1}{9} y_{i \mu}^{3}+\frac{11}{192} y_{i \mu}^{4},
$$

which is exact. This type of analysis leads to

$$
\left\langle M^{(i)}\right\rangle=u \sigma_{3}
$$

and

$$
\ln I_{0}^{\mathrm{tr}}\left(2 \sqrt{y_{i \mu}}\right) \sim M_{\mathrm{B}}^{2}\left(\left(B_{i \mu}^{1}\right)^{2}+\left(B_{i \mu}^{2}\right)^{2}\right)+\text { interaction terms }
$$

with $M_{\mathrm{B}}^{2}=\kappa^{2} u^{2}$, and so

$$
\left\langle\phi_{3}\right\rangle \neq 0 \text {. }
$$

$\left(\right.$ Here $\left.\mathcal{R}_{i \mu}=\cos \left(\left|\underline{B}_{i \mu}\right|\right)+i \underline{\sigma} \cdot \underline{B}_{i \mu} \sin \left(\left|\underline{B}_{i \mu}\right|\right) /\left|\underline{B}_{i \mu}\right|\right)$. Notice that since $u \propto \beta_{1}^{-1}$, in the strong coupling limit $u \rightarrow \infty$. Hence, a parity invariant mass for the fermions is generated dynamically by 
the strongly-coupled $\mathrm{U}(1)$ interactions in $G$ [1], and thus the theory is gapped to charge excitations. But two of the $\mathrm{SU}(2)$ gauge bosons also acquire masses, and hence the $\mathrm{SU}(2)$ group is broken down to $\mathrm{U}_{\tau}(1)$.

To make analytical progress towards an understanding of the confinement properties of spinon and holon degrees of freedom, it is convenient to go to a continuum limit for the low-energy excitations of the theory. By linearizing about a node of the Fermi surface on the underlying theory of fermions we can consider a relativistic field theory of the form

$$
\mathfrak{L}=-\frac{1}{4} F_{\mu \nu}^{a} F^{a \mu \nu}+\frac{1}{2}\left(D_{\mu}^{a b} \phi^{b}\right)^{2}+\mu^{2} \phi^{2}-\lambda\left(\phi^{2}\right)^{2}
$$

where $D_{\mu}^{a b} \phi^{b}=\partial_{\mu} \phi^{a}-i g \epsilon^{a b c} B_{\mu}^{b} \phi^{c}$ and $\mu^{2}>0$, since we have symmetry breaking (actually, in $(2+1)$-dimensions the symmetry broken phase is connected analytically to the phase where the symmetry is unbroken [ [6]). The above form should not be considered as quantitative, but it captures correctly the qualitative features of our approach, and it is sufficient for our purposes here. Terms involving $\mathcal{A}$ and $\mathcal{S}$ are not important for our analysis.

At the perturbative level $B_{3 \mu}$ is massless, and in fact the theory is superconducting [1]. However this is not true when non-perturbative effects are taken into account, such as monopoles, which are instantons in the (2+1)-dimensional theory [7]. The monopole is a Euclidean configuration which behaves asymptotically as

$$
\hat{\phi}^{a}=\hat{r}^{a}, \quad \tilde{F}_{\mu}^{a}(x)=\frac{1}{g} \frac{\hat{r}^{a} \hat{r}_{\mu}}{r^{2}}
$$

where the caret indicates a unit vector and the tilde indicates the dual field tensor.

At the perturbative level the current $J^{\mu}$ associated with $\mathrm{U}_{\tau}(1)$ is conserved and in general is given by

$$
J^{\mu}=\frac{1}{g} \epsilon^{\mu \nu \lambda} \partial_{\nu}\left(\tilde{F}_{\lambda}^{a} \hat{\phi}^{a}\right)
$$

In the context of the model (25), it has been shown [6] that there exists a local gauge invariant field $V(x)$ such that

$$
J^{\mu}=-\frac{i}{4 \pi} \epsilon^{\mu \nu \lambda} \partial_{\mu}\left(V^{*} \partial_{\lambda} V-\text { c.c. }\right) .
$$

The field $V$ interpolates between defect structures and is a disorder variable. In particular we can identify

$$
V^{*} \partial_{\lambda} V-c . c .=\frac{4 \pi i}{g} \tilde{F}_{\lambda}^{a} \hat{\phi}^{a} .
$$

At the perturbative level the current $\tilde{F}_{\mu}$ is given by

$$
\tilde{F}_{\mu}=\tilde{F}_{\mu} \hat{\phi}^{a}-\frac{1}{g} \epsilon^{\mu \nu \lambda} \epsilon^{a b c} \hat{\phi}^{a}\left(D_{\nu} \hat{\phi}\right)^{b}\left(D_{\lambda} \hat{\phi}\right)^{c}
$$

and is conserved. However in the presence of monopoles

$$
\partial^{\mu} \tilde{F}_{\mu}=\frac{4 \pi}{g} \delta^{(3)}(x)
$$

and conservation is lost; the magnetic flux

$$
\Phi=\int d^{3} x \tilde{F}_{0}(x)
$$


does not generate symmetries of the system, i.e. $U_{\alpha}=\exp (i \alpha \Phi)$ is not a symmetry of the physical Hilbert space for arbitrary $\alpha$. Since only configurations with an integer number of monopoles have finite energy the discrete flux transformations

$$
U_{k}=\exp \left(\frac{i g}{2} k \Phi\right)
$$

where $k$ is an integer are still symmetries [6]. Since the fundamental flux is in units of $2 \pi / g$ the only independent operators on the Hilbert space are $U_{0}=\mathbf{1}$ and $U_{1}$ :

$$
U_{0} V(x) U_{0}^{-1}=V(x) \quad U_{1} V(x) U_{1}^{-1}=-V(x)
$$

and so the theory retains a $\mathbb{Z}_{2}$ symmetry. The effective Lagrangian for $V$ is [6]

$$
\mathfrak{L}^{\text {dual }}=\partial_{\mu} V^{*} \partial^{\mu} V-\lambda\left(V^{*} V-\mu^{2}\right)^{2}-\frac{m^{2}}{4}\left(V^{2}+V^{* 2}\right)+h\left(\epsilon^{\mu \nu \lambda} \partial_{\nu} V^{*} \partial_{\lambda} V\right)^{2} .
$$

The parameters appearing in $\mathfrak{L}^{\text {dual }}$ can be calculated perturbatively as

$$
\begin{aligned}
& \mu^{2}=\frac{g^{2}}{8 \pi^{2}}, \lambda=\frac{2 \pi^{2} M_{\phi}^{2}}{e^{2}}, h \propto \frac{M_{\mathrm{B}}}{g^{4} M_{\phi}^{2}}, \\
& m=m_{\mathrm{ph}} \propto e^{-S_{0} / 2} \propto M_{\mathrm{B}}^{2} \exp \left(-M_{\mathrm{B}} / g^{2}\right),
\end{aligned}
$$

where $S_{0}$ is the instanton action. At low energies the derivative term can be ignored. The mass $M_{\phi}$ is that associated with the $\phi$ fields from the Higgs mechanism; in our strong-coupling U(1) situation $M_{\phi} \gg m_{p h}$ [1]. Notice that the presence of a small 'photon' mass $m_{p h}$ implies a pseudogap phase for the statistical model. Because of the $\mathbb{Z}_{2}$ symmetry, when external (adjoint) charges are introduced into the theory, narrow string-like structures are produced [6]. It is tempting to conjecture that such structures are related to stripe phases in the cuprates. The width of the stripes in our model is of order [6] $1 / m_{p h}$, which is finite in underdoped situations. The mass $m_{p h} \rightarrow 0$ as superconductivity is approached, and the stripes will become very wide and eventually occupy the entire space ('absence of stripes'). Because in equation (29) there is the unit vector $\hat{\phi}^{a}$, which is related to a bilinear in the holon fields, the string-like object requires holons. Away from the strip region the Wilson loop shows an area law which is consistent with antiferromagnetic order. This is related to the fact that, as we shall discuss below, the spinon excitations are in the fundamental representation of the $\mathrm{SU}(2)$ group, and as such they are directly associated with the Wilson loop which gives the energy of a state with two heavy external charges in the fundamental representation. A detailed correspondence requires further research. It should be stressed, though, that the above dual description provides a natural and clear picture for the confinement of both holons and spinons. For details we refer the reader to the discussion in ref. [6], which parallels our case here.

We would now like to discuss the effects and (confinement) properties of spinons, $z_{\alpha}, \alpha \in\{1,2\}$. From the construction of the ansatz (4) it becomes clear that the spinon sector in our theory consists of a complex scalar doublet, in the fundamental representation of the $\mathrm{SU}(2)$ gauge group. In the terminology of ref. [6], this situtation corresponds to including scalar constituent 'quarks' $z^{A}$, where $A \in\{1,2\}$ is an $\mathrm{SU}(2)$ fundamental representation index. The presence of such constituents leads to the presence of 'baryons' in our picture. In other words, in our model holon composites correspond to 'mesons', whilst spinon composites correspond to 'baryons'. These are the physical excitations of our spin-charge separated non-Abelian gauge model for the planar doped antiferromagnet. In the dual picture, the presence of 'baryons' is described [6] by the introduction of an additional field $W$, which should be such that : (i) the elementary defect (soliton) of the field $V$ should carry 'baryon' number $1 / 2$ (in the $\mathrm{SU}(2)$ theory) in order to represent the "constituent (fundamental) quark". For this it is necessary that in the core of the defect (where $V$ vanishes) the field $W$ has a non-zero value, whilst outside the core $W \rightarrow\langle W\rangle=0$, since 'baryon' number symmetry cannot be broken spontaneously. (ii) The interaction potential should favour 
configurations in which, for $V$ close to its vacuum expectation value, $W$ is small, whereas for small $V, W$ is non zero. This can be easily implemented by either imposing the $\sigma$-model constraint $V^{*} V+W^{*} W=1 / 8 \pi^{2} \beta_{2}$, or adding the interaction term $\lambda\left(V^{*} V+W^{*} W-1 / 8 \pi^{2} \beta_{2}\right)^{2}$ in the Lagrangian. For convenience we choose the $\sigma$-model constraint. The long-distance properties, we are interested in, are indistinguishable between the two cases [6]. (iii) The 'baryon' should be attached to the topological soliton, so three-derivative terms are necessary in the dual effective Lagrangian, which on symmetry grounds has the form [6]:

$$
\begin{gathered}
\mathfrak{L}^{\text {dual } / \text { baryon }}=\partial_{\mu} V^{*} \partial^{\mu} V+\partial_{\mu} W^{*} \partial^{\mu} W+h\left(V+V^{*}\right)+ \\
+\frac{1}{4 W^{*} W}\left(W^{*} \partial_{\mu} W-W \partial_{\mu} W^{*}\right)^{2}- \\
-\frac{1}{8 \pi\left(W^{*} W\right)\left(V^{*} V\right)} \epsilon^{\mu \nu \lambda}\left(W^{*} \partial_{\mu} W-W \partial_{\mu} W^{*}\right) \partial_{\nu}\left(V^{*} \partial_{\lambda} V-V \partial_{\lambda} V^{*}\right)
\end{gathered}
$$

We now remark that it is an exclusive feature of the $\mathrm{SU}(2)$ case that the vacuum in the one-soliton sector is degenerate [6], corresponding to solitons with 'baryon' number $1 / 2$ and $-1 / 2$. This degeneracy corresponds to an additional global SU(2) 'custodial' symmetry [6], under which the $Z$-matrix in the ansatz (位) is transformed as $Z \rightarrow Z U$. The 'baryon' number is therefore associated with one of the generators of the custodial $\mathrm{SU}(2)$. The fermion matrix is neutral under the custodial symmetry. In the condensed-matter framework this symmetry may be associated with the global $\mathrm{SU}(2)$ spin group of the electron constituents mentioned earlier. Evidently, 'particles' in this dual picture come in pairs with opposite 'baryon' number. In the dual picture the confinement of spinons is evident [6], as can be demonstrated by means of the area law behaviour of the Wilson loop. This completes our qualitative discussion on the symmetry-breaking and confinement properties of our (zero- (or low-) temperature) effective spin-charge separated theory. More work is clearly needed for a quantitative analysis.

\section{Acknowledgements}

We thank A. Kovner and A. Campbell-Smith for discussions. This work is supported in part by the Leverhulme Trust; N.E.M. is also partially supported by P.P.A.R.C. (U.K.).

\section{References}

[1] K. Farakos and N.E. Mavromatos, Phys. Rev. B57, 3017 (1998); Mod. Phys. Lett. A13 1019 (1998).

[2] N.E. Mavromatos and Sarben Sarkar, cond-mat/9912323, Physical Review B (2000), in press.

[3] I. Affleck, Z. Zou, T. Hsu and P.W. Anderson, Phys. Rev. B38 (1988), 745.

[4] N. Dorey and N.E. Mavromatos, Phys. Lett. B 250107 (1990).

Z.Y. Weng, D.N. Sheng, Y.-C. Chen and C.S. Ting, Phys. Rev. B 553894 (1997).

[5] S. Elitzur, Phys. Rev. D12 3978.

[6] A. Kovner and B. Rosenstein, Int. J. Mod. Phys. A8 5575 (1993); JHEP 9809003 (1998).

[7] A.M. Polyakov, Phys. Lett. 59B, 82 (1975); Nucl. Phys. B120, 429 (1977). 